\title{
Joven diáspora marroquí y usos mediáticos. Estudio del proceso de adaptación post-migratoria en España
}

institucional.us.es/ambitos/

José Carlos Sendín Gutiérrez

Universidad Rey Juan Carlos

josecarlos.sendin@urjc.es

\section{Resumen}

El objetivo de este artículo es analizar los procesos de adaptación de la población joven marroquí, a través de sus usos mediáticos en un contexto de ralentización y estabilización de los flujos migratorios en España. El acceso a los medios de comunicación es un factor importante para entender los procesos de adaptación en la nueva sociedad. En este sentido, los medios de comunicación ofrecen a las familias migrantes oportunidades para conocer de primera mano la sociedad de acogida, sin perder la conexión con la sociedad de origen; $y$, al mismo tiempo, constituyen una plataforma para la expresión de sus propias experiencias y puntos

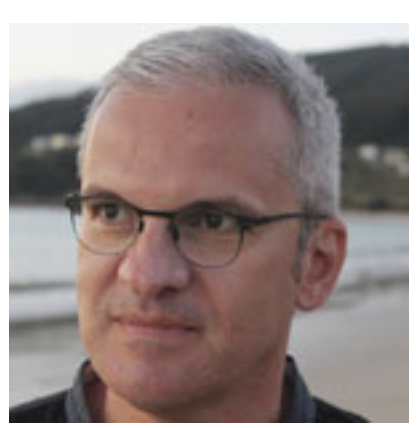

de vista. Partiendo de una revisión crítica de las concepciones tradicionales de integración, el artículo se fundamenta en la noción de diáspora. Se aplica un cuestionario sobre una muestra de 40 jóvenes marroquíes y de otras nacionalidades del mundo árabe, con el fin de conocer sus trayectorias personales y usos mediáticos. Los resultados muestran el papel central de los medios en los procesos vitales de los jóvenes, así como la conformación de múltiples itinerarios personales que se configuran en torno a una idea de diáspora transnacional, que conecta la comunidad de origen, la sociedad de acogida y la comunidad árabe imaginada.

\section{Palabras clave}

Jóvenes marroquíes, medios de comunicación, diáspora, migración.

The aim of this paper is to analyze the processes of adaptation of Moroccan young people through their media

uses in a context of slowing and stabilization of migration flows to Spain. Access to the media is an important factor to understand the processes of adaptation to the new society. In this sense, the media offer opportunities to migrant families to learn about the host society, without losing the connection with the society of origin, which at the same time provides a platform for the expression of their own experiences and views. Based on a critical review of the traditional concepts of integration, the article is focused on the notion of diaspora. A questionnaire was applied to a sample of 40 young Moroccans and other Arab nationals, in order to know their personal paths

and media uses. The results show the central role of the media in their life curse, together with the idea of multiple personal itineraries built around the transnational diaspora conciousness, which connects the community of origin, the host society and the imagined Arab community.

\section{Keywords}

Young Moroccans, media, diaspora, migration.

\section{INTRODUCTION (1)}

Los datos más recientes apuntan a una estabilización, si no paralización de los flujos migratorios hacia nuestro país, que permiten vaticinar que la explosión migratoria ha tocado a su fin (D 'Ancona y Vallés, 2010; Reher y Requena, 2009). Otros autores se han referido a este mismo reducción de 216.125 en 2012, que ya se había apuntado en 2011 con 15.229 menos. Se confirma, por tanto, la 
tendencia a la reducción en el número de extranjeros, lo que también afecta al conjunto de la población empadronada, que se reduce por primera vez desde 1998.

Esta tendencia de fin de ciclo de aumento de la inmigración y, por el contrario, aumento de la emigración de la población autóctona, parece propicia para analizar con más quietud los procesos de adaptación de colectivos de población con amplio arraigo en España, como el marroquí, y hacerlo desde el punto de vista de los usos mediáticos. Hay una larga tradición que explica la importancia del estudio de los medios en el día a día de las personas, en la medida que los medios no sólo son parte de nuestro mobiliario familiar básico (Silverstone, 1999; Livingstone, 2002), sino apéndices de nuestro cuerpo. Sin embargo, hay pocos estudios que se centren en conocer el papel de los medios de comunicación en el proceso de adaptación y gestión de la identidad diaspórica transnacional de la población joven, la denominada comunicación trasnacional (Cogo, Gutiérrez, Huertas, 2008). El concepto transnacional tiene conexiones directas con las migraciones, en el sentido de que permite a las personas acceder a cualquier contenido mediático, bien del país de origen o en el país de acogida; pero además, el espacio transnacional incluye una serie de redes sociales interconectadas, que sirven para intercambiar y transformar tanto ideas, prácticas como recursos (Levitt y Glick Schiller, 2004).

\section{INMIGRANTES MARROQUIEES O DIÁSPORA MARROQUÍ}

Referirse a los marroquíes como inmigrantes o extranjeros, como hacen a efectos prácticos los informes del INE, no deja de ser paradójico en la medida que la historia del flujo de intercambio con España se remonta más allá de la década de los 80 y la primera ola de intensificación de la emigración marroquí data de 1960 (López y Berriane, 2004:51). De hecho, los marroquíes constituyen el segundo colectivo en nuestro país, con un total de 835.188 residentes legales, después del rumano. Hay tres características que singularizan al colectivo marroquí. En primer lugar, su larga trayectoria de presencia en España, especialmente teniendo en cuenta nuestra común historia colonial. En segundo lugar, se encuentra muy extendido a lo largo del territorio español -154 municipios, de los 8.115 que existen en España, tienen más de 1.000 marroquíes empadronados-. Y, por último, muestra unos evidentes indicadores de asentamiento -feminización, nacionalización, segundas generaciones e iniciativas asociativas- (Huertas et al., 2013: 9).

En efecto, el mismo término inmigrante refleja y reproduce, de alguna forma, cierta compartimentación política en Europa que alberga ideologías de exclusión basadas en la diferencia cultural. De esta forma, la palabra inmigrante es una construcción verbal y discursiva que refleja la oposición entre Europa y los Otros, en la misma dirección que Foucault define discurso; es decir, el discurso construye el tema del que se habla, define y produce los objetos de nuestro conocimiento y, por tanto, gobierna la forma en que el tema -la inmigración en nuestro caso- es hablada y razonada. En última instancia, el discurso influencia cómo las ideas se transforman en acciones prácticas y cómo son usadas para controlar la conducta de los demás (Hall, 1997:44). El hecho de que inmigración aparezca casi siempre unida a binomios como "problema de la inmigración" o "crisis de la inmigración", así como su extenso corolario teñido de negatividad (sin papeles, avalancha, mafias, etc) expresa bien cómo el término convierte la migración en una patología social. El problema de fondo de este tipo de discurso problematizador de la inmigración es que no sólo niega a los individuos su posibilidad de viajar a lo largo del tiempo y el espacio, sino que impide observar la historia de asentamiento, las trayectorias de inclusión o exclusión en lugares específicos; en definitiva, oculta el hecho de que las experiencias diaspóricas transnacionales conforman identidades móviles y, al mismo tiempo, identidades nacionales, minoritarias o mayoritarias (Georgiou, 2005, 16).

Desde otras perspectivas, se ha presentado los procesos de adaptación asociados a la emigración como una serie de fases fijas y lineales que no permiten apreciar la rica diversidad y especificidad cultural de los inmigrantes transnacionales no europeos. Por ejemplo, desde la perspectiva de los psicólogos transculturales (Berry, 1997; 1998), se ha propuesto analizar los procesos de aculturación y el estrés asociado a la adaptación a un nuevo entorno, como un proceso que conlleva cuatro posibles estrategias. En primer lugar, la asimilación, en la que las personas deciden no mantener su identidad cultural propia y buscan, por el contrario, la interacción y asimilación con el grupo dominante. En segundo lugar, la integración, definida por esta corriente como la opción elegida por personas que buscan tanto el contacto con sus iguales étnicos, así como con miembros del grupo dominante. En tercer lugar, separación o segregación, que se produce cuando las personas evitan el contacto con el grupo dominante y sólo valoran los contactos con personas de la misma cultura u 
origen. Y, en cuarto lugar, la marginalización que es definida como la propia de aquellas personas que pierden contacto tanto con la cultura de origen como con la cultura y sociedad de acogida. (Bhatia y Ram, 2009).

La movilidad transnacional está dando lugar a diferentes desarrollos teóricos para analizar las transformaciones sociales a que da lugar; es tal su velocidad e intensidad que da lugar a una redefinición incluso del concepto de cultura a nivel global (Appadurai, 1998). Pero también a una reformulación del concepto de familia, las familias transnacionales, que se definen no en términos de proximidad geográfica, sino por las redes de relaciones y sentimientos de identidad colectiva (Benítez, 2011). Estas interacciones por encima de las fronteras llegan a cuestionar las bases sobre las que se asienta el estado nación, así como las ideas preconcebidas sobre la identidad. A pesar de ello, otros autores han desarrollado el concepto de nacionalismo de larga distancia ( Anderson, 1998), que describe cómo los avances en las tecnologías de la comunicación en el mundo occidental permite a los emigrantes enviar dinero, así como hacer circular propaganda para influir en momentos políticos en los países de origen, mientras permanecen protegidos en los países de destino.

Existe una base consolidada de trabajos en nuestro país que analiza la relación entre procesos migratorios y medios de comunicación (Bañón, 2002; Contreras, González y Sierra, 2003; Lario, 2006; Zapata y van Dijk, 2007); así como el tratamiento de la inmigración en los medios (Igartua, Muñiz, Otero y de la Fuente, 2007; Igartua, Muñiz, Otero, Cheng y Gómez, 2008; Lacalle, 2008; Lorite, 2004; Retis y García, 2010; Checa y Arjona, 2011;), y el fenómenos de los medios para y por los inmigrantes, tanto a nivel global (Díaz Nosty, 2007; López, 2007), como dentro de nuestras fronteras (Rigoni y Navarro, 2007; López Romero, 2009; VV.AA, 2006, 2007 y 2008; Gómez-Escalonilla, 2008). Sin embargo, son más escasos los trabajos dedicados a analizar la relación entre medios de comunicación y migrantes marroquíes o árabes (Amezaga, 2001; Huertas, Reguero y Sagarzazu, 2010; Benítez Eyzaguirre, 2011), y especialmente, si nos centramos en la población joven de estos países.

Estos últimos trabajos que relacionan a los migrantes marroquíes con los medios destacan la importancia del consumo de los medios del país de origen, en especial la televisión, también apuntada en trabajos anteriores (Amezaga et al., 2001), como instrumento de comunicación transnacional para mantener las raíces culturales y el contacto con la comunidad de origen. También se destaca la brecha de género a la hora de acceder a los medios y, especialmente, en la integración social, tendencia que se acentúa en las mujeres de la primera generación (Huertas, Reguero y Sagarzazu, 2010: 225,229).

Otro trabajos realizados en Marruecos se han centrado en la influencia del consumo mediático, especialmente televisivo en los proyectos migratorios de la población. Así, se ha destacado el diferente papel desempeñado por la televisión autóctona, que a pesar de servir de referencia principal es objeto de crítica. Mientras, la televisión panárabe, Aljazeera, contribuye a alimentar una identidad común que supera las fronteras de los países árabes. Por su parte, las televisiones occidentales, en particular la española, ejerce su influencia no sólo en patrones identitarios asociados a la vestimenta (aunque sea de forma crítica), sino especialmente en las expectativas de mejora y proyectos de movilidad (Benítez Eyzaguirre, 2011: 85).

Trabajos internacionales sobre colectivos inmigrantes jóvenes (ex Unión Soviética e Israel) apuntan algunas características del consumo de medios tradicionales en la diáspora, así como las interrelaciones familiares. De esta forma, los jóvenes parecen rechazar la presión paterna para ver la televisión del país de origen, puesto que la perciben como un obstáculo para su adaptación a la sociedad de acogida, mientras que los padres insistirían en consumir televisión autóctona desde la diáspora como una herramienta para que los hijos mantengan la identidad original. Esta presión paterna se traduciría en que muchos jóvenes inmigrantes participan en lo que se ha denominado "cultura de domingo", para unirse a sus padres en el visionado de algún programa de la televisión del país de origen (Elias y Lemish, 2008:23).

En otras investigaciones se mencionan dos roles principales desempeñados por los medios: preservación cultural y alivio del sentimiento de nostalgia, al tiempo que herramienta para ayudar a los jóvenes en su proceso de adaptación. Por su parte, las familias inmigrantes emplearían los medios en dos direcciones, con un papel destacado por parte de los padres. En primer lugar, un conjunto de padres intentan controlar el consumo de medios de sus hijos, ya que parten de una supuesta influencia negativa por parte de los medios; sin embargo, otros padres difieren en sus estrategias para facilitar tanto el contacto con la cultura de origen como la 
necesaria adaptación de los hijos a la cultura de acogida. Así, encontramos las dos estrategias familiares combinadas: muchas familias intentan recrear la atmósfera cultural familiar por medio de un alto consumo de canales de televisión vía satélite; mientras que otras familias evitan instalar la televisión por satélite, para evitar entorpecer en sus hijos el proceso de adquisición de la lengua de acogida (Christopoulou y de Leeuw, 2004).

Por lo que respecta al uso de Internet por los jóvenes inmigrantes en la diáspora, las investigaciones realizadas en la materia apuntan a que Internet tiende a reemplazar a los medios tradicionales en el proceso de adaptación social de los jóvenes. Esto es así por las características de Internet: accesibilidad, interactividad y anonimato. Además, permite realizar contactos interpersonales entre jóvenes de la misma procedencia y que experimentan el mismo estrés de adaptación a la nueva cultura; de esta forma, Internet no sólo ayudaría a superar los períodos de soledad asociados a la primera fase de llegada, sino que contribuiría a conformar redes virtuales de apoyo entre jóvenes inmigrantes, en un momento en que deben superar dos retos fundamentales en sus vidas: adolescencia y reubicación (Elias and Lemish, 2009:547). Internet proporciona un espacio vital a las comunidades de inmigrantes en Reino Unido, que sirve para compartir información y como foro para la discusión pública en asuntos relacionados con la exclusión de las minorías en los medios generalistas, como la BBC (Georgiou, 2006b).

Finalmente, en este trabajo pretendemos incidir en la línea de los estudios que están analizando el papel de los medios de comunicación en el complejo proceso de construcción de las identidades diaspóricas y, en este caso, en el contexto de sociedades post-migratorias, como es el que parece caracterizar en estos momentos a España. El término diáspora tiene la ventaja de que frente a otros esquemas simplificadores y lineales, permite aprehender mejor la situación de los migrantes. Comunidades diaspóricas son aquellas que intentan mantener conexiones y compromisos -reales o imaginarios- con su país de origen y se reconocen a ellos/as mismos como una comunidad colectiva. Por esta razón, personas que únicamente viven fuera de sus comunidades de origen no forman automáticamente una diáspora (Tölöyan, 1996). Las diásporas se generan cuando comunidades de emigrantes no encuentran representada su cultura en la sociedad de acogida y comprueban el silenciamiento y ocultamiento de su propia tradición cultural. Esta sensación es experimentada de forma aguda por las comunidades no europeas y no blancas, que deben renegociar constantemente conceptos como aquí y allí, pasado y presente, país de origen y país de acogida, yo y el otro. Por esta razón, el concepto de diáspora permite pensar los procesos de aculturación e identidad de forma flexible y plástica.

\section{METODOLOGÍA}

La técnica de investigación utilizada en coherencia con el objetivo plateado - analizar los procesos de adaptación y configuración identitaria de la población joven marroquí, a través de sus usos mediáticos- fue la entrevista semi-estructurada con jóvenes marroquíes y de otras nacionalidades árabes. Se empleó para ello un cuestionario diseñado y testado en un proyecto anterior (Ponte y Simões, 2012) y se aplicó a una muestra compuesta por 40 jóvenes, con edades comprendidas entre los 16 y los 39 años, mitad mujeres y mitad hombres. También se realizó una entrevista adicional con una familia compuesta por siete miembros.

Las entrevistas se realizaron entre enero y abril de 2012 en varias localidades del suroeste de Madrid, en especial Fuenlabrada. Esta ciudad del corredor sur de la Comunidad de Madrid, concentra la mayor parte de la población marroquí -91.022 personas- de toda la región. Se trató de incluir jóvenes de diferentes condiciones socioeconómicas. El cuestionario estaba compuesto por dos bloques temáticos: datos personales e historia de vida, y usos familiares de los medios de comunicación y usos personales actuales. Las entrevistas fueron grabadas y transcritas íntegramente, para ser analizadas conjuntamente con las notas recopiladas durante y después de cada entrevista.

Las entrevistas duraron aproximadamente 40 minutos, a excepción de la realizada a la familia que duró más de 1 hora y media. Se llevaron a cabo con el apoyo de un asistente de investigación de origen marroquí, competente en árabe y castellano. La selección de los entrevistados se realizó a partir de la mezquita de Fuenlabrada, aplicando el procedimiento bola de nieve para identificar diferentes lugares: Centro Cultural Islámico de Fuenlabrada, Universidad Politécnica y Universidad Rey Juan Carlos, locutorio de Fuenlabrada, Asociación marroquí de Getafe, Asociación cultural árabe Tayeba, Asociación Centro Trama y en la casa de los encuestados en el caso de la familia. 


\section{RESULTADOS}

\section{Historia de vida: razones para emigrar y su vida en el extranjero.}

El uso de los medios no se produce en el vacío, sino que se da en contextos personales, a veces de ruptura y desorientación. Este apartado nos permite comprender cómo se vive la realidad del cambio de entorno cultural e idiomático.

Las razones para migrar son múltiples, a pesar de la idea predominante que relaciona emigración con búsqueda de empleo. Algunos vinieron para mejorar o avanzar en sus estudios, otros llegaron en busca de un trabajo, mientras que en otros casos se unen a sus familias que ya están en España. Una sensación compartida por los entrevistados en su etapa inicial fue la impotencia y la desorientación debido al desconocimiento de la lengua, así como los problemas para interpretar los códigos locales de comportamiento. Esta experiencia se ha identificado igualmente en otros estudios, a pesar de que analizaban otros colectivos migratorios (Elias y Lemish, 2009:540)

Una vez en España las familias incorporan sus tradiciones y fiestas en el nuevo contexto. Sin embargo encontramos diferentes percepciones, de acuerdo con las distintas generaciones:

Madre de familia, 39 años:

"Para mí, hay algunas diferencias en la celebración de las fiestas entre Marruecos y aquí. Aquí no tengo a toda mi familia, así que no soy capaz de celebrar la fiesta del cordero con mi madre o hermanos, además de las dificultades a encontrar un cordero y matarlo durante este mes, ya que está prohibido hacerlo en casa".

Chica, 22 años.

"Las fiestas que celebramos son las típicas familiares y religiosas, como el Ramadán. Creo que no hay diferencia entre celebrar en Marruecos y aquí, es lo mismo."

Chica, 29 años.

"Hacemos celebraciones religiosas, así como fiestas nacionales. La celebración aquí es mejor que en Marruecos, porque en mi país sólo los hombres pueden asistir a la mezquita durante el Ramadán, mientras que, por el contrario, aquí vamos todos a la Mezquita, mujeres, niños, etc".

Encontramos diferentes patrones respecto a los usos de la lengua árabe y la española, también de acuerdo a las generaciones. Mientras que los padres parecen utilizar el árabe como principal vía de comunicación, al tiempo que como instrumento del mantenimiento de una identidad musulmana; la generación más joven usa tanto el árabe como en español.

Chico, 16 años, (nacionalidad española).

"He venido a España con tres meses, mi vida está en España...En mi país y con mis padres hablamos el dialecto marroquí, porque no conocen el idioma español, pero mis hermanos y yo hablamos español".

Los adultos parecen representar el papel de puente entre las dos sociedades. Intentan luchar por mantener las raíces a través de uso de la lengua, las costumbres y la religión. Hacen esto al mismo tiempo que reconocen la importancia de involucrarse en el nuevo entorno, por lo que el idioma del país de asentamiento se considera relevante, en especial por razones prácticas.

Madre de familia, 39 años:

"En casa hablamos el dialecto marroquí, porque es muy importante para nosotros, especialmente para mis hijos, porque cuando viajamos a Marruecos cómo pueden comunicarse con sus familiares si no conocen el dialecto marroquí? Es importante no perder nuestras raíces... Tengo cuatro hijos que estudian árabe en la mezquita, porque sin leer ni escribir en árabe ¿cómo se puede leer el Corán?" 
"Yo creo que si alguien no habla español no puede participar activamente en la vida diaria. Por ejemplo, las mujeres que no hablan español no salen. Es importante hablar español para ir al médico o ir a lugares oficiales".

El sentimiento híbrido de pertenencia aparece repetidamente, al mismo tiempo que el choque cultural. Así, para algunos de los encuestados España es sólo un lugar temporal para estudiar y trabajar, porque el lugar ideal está al otro lado del Estrecho, en Marruecos. En otros casos, muestran un mayor grado de adaptación, aunque expresan sus problemas en la búsqueda de un trabajo y las dificultades que encuentran por llevar el hiyab (velo). Como señala Máriam Martínez en muchas sociedades europeas, los problemas relativos a la libertad de expresión religiosa, en la que el uso del velo en lugares públicos alimenta un debate político y mediático viciado, han conseguido invisibilizar otros temas "relativos a la justicia de género, como son la división sexual del trabajo, los procesos de socialización de género y el establecimiento de normas y pautas sociales hegemónicas, que suelen construir jerarquías de poder siguiendo roles de género" (2011:40).

Chico, 16 años, (nacionalidad española).

"Ahora estoy bien, no quiero volver a Marruecos. Pero cuando sea mayor espero volver, porque España no es mi país. Ahora, como soy joven, tengo que estudiar y trabajar..."

Chica, 24 años (nacionalidad Siria).

"Es difícil encontrar un trabajo si estás usando el hiyab. He hecho muchas entrevistas de trabajo y me dijeron que sólo puedo trabajar como secretaria, pero sin hiyab. No puedo trabajar sin mi hiyab. Esto me influye negativamente".

\section{Usos familiares de los medios de comunicación y usos personales actuales.}

El medio de comunicación familiar por excelencia es la televisión. La dieta televisiva se configura por una mezcla de canales marroquíes como Al Aoula TV, Almaghribia TV y 2M Maroc, y trasnacionales como Aljazeera, a los que acceden a través de los satélites Nilsat, Arabsat y Hotbird. Y, por último, los canales de televisión españoles generalistas. La mayoría de los encuestados se refieren a Almaghribia TV y a Aljazeera como los principales canales de noticias, pero a Aljazeera como uno de los medios más importantes para mantenerse informados sobre los acontecimientos relacionados con la primavera árabe. Los canales de televisión en España son utilizados para saber lo que ocurre, aprender español y entretenerse.

La valoración de la televisión es múltiple, en la misma línea que los hallazgos mostrados en otros estudios (Benítez Eyzaguirre, 2011); es muy apreciada la transnacional, mientras que la del país de origen es criticada por ser poco crítica y plural; y, por último, la española es apreciada en lo que supone herramienta para conocer el contexto social de destino, pero criticada por su poca calidad, politización y por su etnocentrismo, expresado en la ausencia de contenidos -generalistas y religiosos- en lengua árabe.

Por el contrario, los medios impresos son menos populares entre las familias, a excepción de los diarios gratuitos que ojean en el Metro. Internet es mencionada por todos los entrevistados como la plataforma preferida, por ser polivalente, flexible y ampliamente accesible.

Esta valoración de Internet, al igual que la televisión, en tanto que agentes de socialización en las etapas iniciales de llegada a la sociedad de acogida se puede apreciar claramente. Los encuestados de la segunda generación, ya nacidos en España, utilizan prioritariamente canales españoles, porque entienden peor el árabe.

Chica, 20 años.

"Mi medios de comunicación preferido es Internet, ya que ofrece la forma más fácil de hablar con la gente.

Puedo usarlo para trabajar, estudiar y para hablar con mis amigos".

Chica, 21 años.

“En casa vemos siempre la televisión. Me gustan las noticias y veo las imágenes completas...También la 
televisión es mi mejor entretenimiento".

Chica, 28 años.

"Prefiero Aljazeera, porque siempre dice lo que es; sin embargo, mientras que 2 M TV y Al Aoula TV no presentan la realidad de los hechos. Espero que el nuevo ministro del gobierno actual cambie la situación de los medios de comunicación marroquíes".

Chico, 25 años.

"El medio de comunicación que veía cuando se inicio la revuelta en el mundo árabe era Aljazeera, porque puedo confiar en ellos. Aljazeera da una buena cobertura de lo que sucede en el mundo árabe, ya que tiene la capacidad de tener enviados especiales".

Chica (nacionalidad siria).

"Echo de menos un canal en lengua árabe en la televisión española. Esto es un obstáculo para muchas personas árabes que no hablan español. Cuando llegué a España empecé a ver las noticias del canal $24 \mathrm{hrs}$ (TVE), para aprender español".

Chico. 24 años.

"España no es una sociedad musulmana, por lo que es difícil encontrar programas religiosos en la televisión para aprender la cultura islámica. Mi opinión sobre los medios de comunicación españoles es que están controlados por partidos políticos, ya sea de izquierda o derecha, sobre todo en las noticias. No hay ninguna manera de saber si son imparciales".

Chica, 22 años.

"Yo veo las cadenas como Cuatro, La2, otras cadenas me parecen basura. Yo no veo programas árabes porque no entiendo el árabe, soy una musulmana española típica".

Como se ha mencionado, Internet es la plataforma preferida por todos los encuestados y constituye parte del ecosistema personal para acceder a la información que les interesa, tanto de la sociedad de origen como de la de residencia, así como al ocio y los amigos.

Chico, 23 años.

"Tengo un ordenador y conexión a Internet en casa. Utilizo mucho Internet porque yo no veo la televisión, y también veo los deportes y noticias políticas en general, todo lo que pasa en el mundo".

Chica, 29 años.

"Yo no tengo ordenador ni Internet en casa. Sólo en mi trabajo. Yo lo uso en mi trabajo para servicios como transferencias de dinero".

Chico, 21 años.

"Para saber lo que pasa en Marruecos, yo uso las webs de los periódicos electrónicos, como Hespress, Hibapress, y para saber lo que pasa en mi ciudad de Tánger sigo los medios electrónicos de Tánger, como Tangernews".

Chico, 25 años.

"Sólo uso Internet en el salón de mi casa. Lo uso para ver Youtube, y las noticias de Marruecos, en particular de Tánger".

Chica, 33 años. 
"Tengo un ordenador en casa, y uso Internet para ayudar a mis hijos con los deberes del colegio y otras actividades".

Chica, 13 años.

"Para saber lo que está pasando en mi país, Marruecos, utilizo Facebook, además de para conectar con mis primos. Facebook para mí es mi relación con mi familia, y también uso Tuenti para conectar con mis amigos".

Chico, 14 años.

"Yo Tuenti, Facebook, ya que es el mejor método para conectar con los demás. Busco otras personas como yo, hacer amigos de otros países".

Chico, 26 años.

"Yo uso Internet para saber qué está pasando en mi país, charlar con mis amigos de Marruecos y en el aula. Puedo conectar con ellos a través de Facebook. Facebook para mí es una página que ha cambiado el mundo".

Chico, 30 años (nacionalidad española y origen sirio).

"En la casa tenemos tres ordenadores portátiles. Uso Internet para conectar con mi familia en Siria desde Skype, Facebook, o para llamar a los teléfonos móviles a través de Internet"

Chica, 21 años.

"Facebook para mí es fundamental, porque me puedo comunicar con el resto del mundo. Ha sido especialmente importante durante la primavera árabe".

Chica, 26 años. (nacionalidad española).

"Las redes sociales son una buena forma de transmitir noticias, especialmente Facebook, ya que tengo amigos en Siria, Túnez y Egipto que han estado publicando una gran cantidad de información. Facebook es una oportunidad para la comunicación. Antes era muy crítica hacia Facebook, pero he visto su papel durante la primavera árabe y me di cuenta de su potencial para incrementar el conocimiento, la divulgación, y saber lo que ocurre en Túnez o en Arabia Saudita. Hay muchas noticias que no aparecen en los medios de comunicación españoles".

Los teléfonos móviles son utilizados por los encuestados de mayor edad esencialmente para comunicarse con la familia en Marruecos, mientras que los encuestados más jóvenes lo utilizan para comunicarse con amigos, jugar y escuchar música, siguiendo el mismo patrón que el resto de los adolescentes.

Chico, 31 años.

"Uso el teléfono móvil para hablar con mi familia en Marruecos. Me descargué Skype para hablar con ellos, para no gastar mucho dinero. También tengo un programa en mi ordenador que me permite hablar gratis con teléfonos fijos".

Chico, 26 años,

"Tengo un móvil para acceder a las noticias de la universidad, hablar con mi mujer, que vive en Francia, y también para hablar con mis padres en Marruecos."

Chico, 16 años,

"Yo uso mi teléfono para entretenerme, hablar con mis amigos, escuchar música y tomar fotos ".

\section{CONCLUSIONES}

Este trabajo representa un esfuerzo por acercarse a conocer cómo es experimentado el proceso de adaptación 
a la sociedad de acogida por parte de los jóvenes árabes, e indirectamente al proceso de construcción de la identidad diaspórica, a través del análisis de los usos mediáticos. Todo ello en un contexto de ralentización (o inversión) del flujo inmigratorio en nuestro país.

Una primera constatación, de acuerdo con las opiniones de los participantes en este estudio es el papel predominante de los medios de comunicación en sus vidas diarias y, por tanto, en el proceso de construcción de la identidad diaspórica y el sentido de pertenencia. En este trabajo, la mayoría de los entrevistados muestran un nivel razonable de alfabetización mediática, puesto que acceden a diferentes medios y diferentes plataformas en función de sus intereses y para complementar información.

Tanto los medios de comunicación tradicionales como Internet son ingredientes esenciales para la dieta mediática en el contexto diaspórico. En relación con la televisión, medio de uso generalizado, tanto directamente como a través de Internet, se han detectado diferentes tipos de "menús": 1) televisión del país origen mezclado con televisión trasnacional (Aljazeera); 2) televisión del país de origen con televisión española; y 3) televisión española. La televisión se utiliza, sobre todo por los inmigrantes de primera generación, para conocer el nuevo contexto, aprender el idioma y acostumbrarse al nuevo ambiente; es decir, como herramienta para establecer puentes o cerrar la brecha entre el país de origen y el de destino.

La anterior constatación nos lleva a que el sentido de pertenencia o el complejo proceso de formación de la identidad diaspórica no parecen estar directamente relacionados con la elección exclusiva de los canales de televisión del país de origen, como podría pensarse en un principio. La conciencia diaspórica se forja precisamente en la multiplicidad de opciones que ofrecen las diferentes cadenas y plataformas de acceso a los medios, así como en las diferentes oportunidades y comunidades de destino con las que los participantes se identifican.

Hemos comprobado cómo algunos participantes jóvenes siguen escuchando música clásica árabe y también reclaman más contenidos en este idioma en la televisión local, tendencia especialmente resaltada por la madre de familia, que intenta reforzar el vínculo con la comunidad de origen. Sin embargo, también se conectan a Facebook para hablar con amigos del país de origen o con nuevos contactos en otros países árabes, mientras se informan de los últimos acontecimientos de la primavera árabe y chequean su impacto en el país de origen (Marruecos). Por lo tanto, tratan de reforzar la identidad diaspórica árabe, al mantener los contactos y compromisos (reales y / o imaginarios) con el país de origen, así como reconocerse a sí mismos y actuar como una comunidad colectiva. Todo ello apunta en la dirección de que los medios son usados con finalidades diferentes y sirven como herramienta para articular el sentido de pertenencia entre las tres comunidades imaginadas que aparecen: la de origen, la de destino y la panárabe.

Por último, las personas nos movemos a lo largo de varios espacios de pertenencia, tanto físicos como simbólicos. Sin embargo, no todos los individuos o grupos disfrutan del mismo nivel de movilidad, pues existen importantes restricciones en relación con el género, la edad y la clase social. El incidente mencionado por una de las participantes -ser rechazada en varias entrevistas de trabajo para llevar el velo- pone de relevancia este tipo de limitaciones, en este caso a la movilidad social por medio del acceso al trabajo. De la misma forma, expresa de forma cruda cómo los jóvenes de la diáspora árabe contemporánea viven su proceso de negociación con la cultura de destino; en muchas ocasiones es contradictoria, dinámica y, muchas veces, inestable.

Como hemos comprobado, la adaptación y la integración en la sociedad de acogida, objetivo de las políticas públicas en materia de inmigración, puede ser lograda temporalmente por algunas personas para ser perdida en algún momento del proceso; o puede simplemente no ser una opción para algunos de los entrevistados. Dicho en otros términos, el viaje de la aculturación no supone una trayectoria teleológica que tiene un punto de llegada fijo, sino que se negocia continuamente en procesos vitales que se configuran en el ámbito de lo trasnacional. Por tanto, serán necesarias más investigaciones a futuro que amplíen tanto el número de integrantes en el estudio, como el tiempo; es decir, conformar paneles de poblaciones diaspóricas que permitan conocer mejor el complejo proceso de adaptación y conformación de las identidades transnacionales. 
Aja, E., Arango, J. y Oliver, J. (eds.) (2009) La inmigración en la encrucijada. Anuario de la inmigración en España. Barcelona. Fundación CIDOB.

Amezaga, Jose et al. (2001). "Biladi. Usos de la televisión por satélite entre los y las inmigrantes magrebies en Bilbao". Zer, 10, 81-105.

Anderson, B. (1998). The spectre of comparisons: Nationalism, Southeast Asia and the world. London: Verso. Appadurai, Arjiun (1998) Modernity at Large: Cultural Dimensions of Globalization. Minneapolis: University of Minnesota Press.

Bañón, A. (2002) Discurso e inmigración. Murcia: Universidad de Murcia.

Benítez Eyzaguirre, Lucía (2011) La recepción de la televisión transnacional y los proyectos migratorios: Un estudio de campo en Marruecos. En IC - Revista Científica de Información y Comunicación, 8, pp. 77 - 94.

Benítez, José Luis (2011). La comunicación transnacional de las familias e-migrantes. PNUD/UCA San Salvador.

Berry, J. W. (1997). Immigration, acculturation and adaptation. Applied Psychology: An International Review, 46, $5-68$.

Berry, J. W. (1998). Acculturative stress. In P. B. Organista, K. M. Cren, \& G. Marin (Eds.), Readings in ethnic psychology (pp. 117-122). New York: Routledge.

Cea D’Ancona, Ma Á. y Valles Martínez, M. S. (2010) Evolución del racismo y la xenofobia en España [Informe 2010] Madrid. OBERAXE. Ministerio de Trabajo e Inmigración.

Checa Olmos, Juan Carlos, Ángeles Arjona Garrido (2011). Españoles ante la inmigración: El papel de los medios de comunicación. Comunicar: Revista científica iberoamericana de comunicación y educación, № 37 , (141-149).

Christopoulou, N. and S. de Leeuw (2004) Home is Where the Heart Is: Family Relations of Migrant Children in Media Clubs in Six European Countries. Research report from 'Children in Communication About Migration' (CHICAM). London: Centre for the Study of Children, Youth and Media, Institute of Education, University of London.

Cogo, Denise; Gutiérrez, María; Huertas, Amparo (coord.) (2008), Migraciones transnacionales y medios de comunicación. Relatos desde Barcelona y Porto Alegre. Madrid: Los libros de la Catarata.

Contreras, F.R. González Galiana R. y Sierra Caballero, F. (coords.) (2003) Comunicación, cultura y migración . Sevilla: Editorial Consejería de Gobernación, Junta de Andalucía.

Díaz Nosty, B., 2007: Los medios de comunicación en la experiencia migratoria latinoamericana. Madrid: Fundación Telefónica

Elias, N. and D. Lemish (2008) Media Uses in Immigrant Families: Torn between "Inward" and "Outward" Paths of Integration, International Communication Gazette. 70(1): 23-42.

Elias, Nelly and Dafna Lemish (2009) Spinning the web of identity: the roles of the internet in the lives of immigrant adolescents. New Media Society .Vol 11(4): 533-551

Georgiou, M, (2005): Diasporic Media Across Europe: Multicultural Societies and the Universalism-Particularism Continuum. Journal of Ethnic and Migration Studies, 31 (2), 481-498.

Georgiou, M. (2006b) Diasporic Communities Online: A Bottom-up Experience of Transnationalism, in K. Sarikakis and D. Thussu (eds) The Ideology of the Internet: Concepts, Policies, Uses, (pp. 131-46). Cresskill, NJ: Hampton Press. 
Gomez-Escalonilla, G. (Coord.) (2008): Voces de la inmigración. Medios latinos en Madrid. Madrid: Editorial Universitas.

Hall, Stuart (Ed) (1997) Representation: Cultural representations and signifying practices. London: Sage and The Open University

Huertas, A.; Reguero, N. y Sagarzazu, I. (2010): Integración social y consumo mediático y cultural de los migrantes magrebíes en Cataluña. Zer, 29, 213-233

Huertas, A; Martínez, Y.; Moreras, J. (2013) Prácticas y consumos mediático-culturales del colectivo marroquí en España. Documentos de trabajo 11/2013. Madrid: Fundación Alternativas.

Igartua Perosanz, Juan José; Muñiz Muriel, Carlos; Otero Parra, José Antonio; de la Fuente Juan, Montse. (2007) El tratamiento informativo de la inmigración en los medios de comunicación españoles. Un análisis de contenido desde la Teoría del Framing. Estudios sobre el mensaje periodístico, № 13, (91-110).

Igartua, Juan-José; Muñiz, Carlos; Otero, José; Cheng, Lifen; Gómez-Isla, José. (2008) Recepción e impacto socio-cognitivo de las noticias sobre inmigración. Revista de Psicología Social .23 (1), 3-16.

Lacalle, C. (2008) El discurso televisivo sobre la inmigración. Barcelona: Omega.

Lario Bastida, M., (coord.), (2006): Medios de comunicación e inmigración. Madrid: Convivir sin racismo (CAM, Obra social).

Livingstone, S (2002) Young people and new media. Childhood and the changing media environment. London: Sage.

López García, Bernabé y Berriane, Mohamed (2004) Atlas de la inmigración marroquí en España. Madrid: Ediciones de la Universidad Autónoma de Madrid-Taller de Estudios Internacionales Mediterráneos-Ministerio de Trabajo y Asuntos Sociales.

López, X., (2007): “Un sector en auge. Evolución de la prensa en español en Estados Unidos”. Telos, 70. Cuaderno central: las industrias culturales en español en Estados Unidos.45-97.

López Romero, Laura (2009) Prensa extranjera en España: la integración social a través de los medios escritos. Telos: Cuadernos de comunicación e innovación, №. 80, (116-123).

Lorite García, Nicolás (2004) Cómo tratar la inmigración en los medios pensando en la inteculturalidad. Red digital: Revista de Tecnologías de la Información y Comunicación Educativas, № 5.

Martínez, Máriam (2011) ¿Es el multiculturalismo bueno para los inmigrantes? Reis 135, julio-septiembre 2011, 27-46.

Ponte, C, Simoes, J.A. (2012) Training Graduate Students as Young Researchers to Study Families' Use of Media. Comunicar Revista Científica Iberoamericana de Comunicación y Educación (38): 103-112.

Reher, D. S. y Requena, M. (eds.) (2009) Las múltiples caras de la inmigración en España. Madrid: Alianza Editorial.

Retis, Jessica; García, Paola (2010) Jóvenes inmigrantes latinoamericanos en la prensa española. Narrativas mediáticas de la alteridad: el caso de las violencias urbanas. Revista mexicana de ciencias políticas y sociales . №. 209, 135-160.

Ricard Zapata-Barrero y Teun A. van Dijk (eds.).(2007) Discursos sobre la inmigración en España. Los medios de comunicación, los parlamentos y las administraciones. Barcelona: Fundación Cidob. 2007.

Rigoni, I. y Navarro, L., (2007): “Los medios de comunicación de minorías étnicas" en $2^{\circ}$ Anuario de la Comunicación del Inmigrante en España 07/08. Madrid: Etnia Comunicación 
Silverstone, R. (1999). Why study the media? London: Sage.

Sunil Bhatia, Anjali Ram (2009) Theorizing identity in transnational and diaspora cultures: critical approach to acculturation. International Journal of Intercultural Relations 33. 140-149.

Tölöyan, Khaching (1996). Rethinking diaspora(s): Stateless power in the transnational moment. Diaspora, 5, 335.

VV.AA., (2006, 2007 y 2008): Anuario de la comunicación del inmigrante en España. Madrid: Etnia Comunicación.

\section{Anexo 1}

\begin{tabular}{|l|l|l|l|}
\hline Mujer & Edad & Hombre & Edad \\
\hline 1 & 13 & 0 & 0 \\
\hline 1 & 14 & 2 & 16 \\
\hline 3 & 20 & 1 & 19 \\
\hline 3 & 21 & 2 & 20 \\
\hline 0 & 22 & 0 & 22 \\
\hline 1 & 24 & 3 & 21 \\
\hline 2 & 25 & 1 & 23 \\
\hline 2 & 26 & 4 & 24 \\
\hline 1 & 28 & 2 & 25 \\
\hline 2 & 29 & 1 & 26 \\
\hline 1 & 32 & 1 & 30 \\
\hline 2 & 33 & 2 & 31 \\
\hline 0 & 0 & 1 & 43 \\
\hline Total & & Total & \\
\hline 19 & & 20 & \\
\hline
\end{tabular}

\section{Anexo 2}

\begin{tabular}{|l|l|l|}
\hline - Hombres & Mujeres \\
Tiempo total entrevistas & $5: 15^{\prime}: 18^{\prime \prime}$ & $4: 26^{\prime}: 57^{\prime \prime}$ \\
\hline & & \\
\hline
\end{tabular}


(1) Una primera versión de este trabajo fue presentada en el panel Diaspora, migration and the media. Family, audiencehood, and cultural diversity: conceptual and empirical challenges in changing Europe, en el marco de la conferencia ECREA, celebrada en Estambul, Turquía, el 25 de Octubre de 2012. El panel fue propuesto por los integrantes del grupo de trabajo 4 del proyecto COST Action IS0906 Transforming audiences, Transforming societies, del que el autor forma parte.

(2) http://www.ine.es/prensa/np776.pdf (acceso el 8 de Julio de 2013).

Ámbitos. Revista Internacional de Comunicación, n.26, año 2014, tercer trimestre (otoño). 